\title{
Existence of global strings coupled to gravity
}

\author{
Gary W. Gibbons, Miguel E. Ortiz, and Fernando Ruiz Ruiz* \\ Department of Applied Mathematics and Theoretical Physics, University of Cambridge, 3 Silver Street, \\ Cambridge CB3 9EW, United Kingdom
}

(Received 28 November 1988)

\begin{abstract}
We consider $\sigma$-model strings and $\mathrm{U}(1)$ global strings coupled to gravity and look for static solutions with whole-cylinder symmetry. We prove that in both cases there are no regular spatially compact solutions. The U(1) global string admits no asymptotically well-behaved solutions, whereas $\sigma$-model strings can be constructed under certain assumptions. Finally we present some singular solutions and show that one of them corresponds to a physical global string.
\end{abstract}

\section{INTRODUCTION}

Over the past few years cosmic strings have attracted much attention due to their important role in cosmology. ${ }^{1}$ These strings are expected to form after the breaking of both local and global symmetries in the corresponding field theory. Much progress has been made in the understanding of local strings. Nielsen and Olesen ${ }^{2}$ considered the Abelian Higgs model and constructed a static cylindrically symmetric solution with boost invariance in the axial direction in flat spacetime. Garfinkle ${ }^{3}$ studied the exact gravitational field for such a solution and proved the existence of an asymptotically conical metric with deficit angle $\delta \approx 8 \pi G \mu$, where $\mu$ is the mass per unit length of the string. Laguna-Castillo and Matzner ${ }^{4}$ found a numerical solution to Garfinkle's equations, and Gregory ${ }^{5}$ proved its stability.

The situation for global strings is rather more complicated and requires clarification. Using a scaling argument originally due to Derrick, ${ }^{6}$ one can easily prove that there exist no stable global strings of finite energy per unit length in a flat background. In brief, consider any scalar model with arbitrary non-negative potential. Any static straight string in $3+1$ dimensions would give rise to a static soliton solution in $2+1$ dimensions whose $(2+1)$-dimensional energy $E$ is the energy per unit length of the string. This solution would be an extremum of $E$. Under a scale transformation $x^{\mu} \rightarrow \alpha x^{\mu}, \mu=1,2,3$, the energy goes to $E_{\alpha}=T+\alpha^{2} V$, where $T$ and $V$ are the kinetic and potential energies, both non-negative. Static finiteenergy solutions are thus ruled out since there is no $\alpha \neq 0$ which extremizes $E_{\alpha}$. In fact, it turns out that there do exist solutions representing global strings in flat spacetime, but their energy density falls off as $1 / r$ so that their energy per unit length diverges.

In a cosmological context, one might argue that a spacetime containing a global cosmic string will not be asymptotically flat, and so that there is no necessity for considering only strings of finite energy per unit length. One would still like to know, however, whether or not it is sensible to talk about the gravitational field of an isolated global cosmic string in the same way that one can talk about the asymptotic gravitational field of a local string.
In the latter case an appropriate concept of total energy, including gravitational, is given by Thorne's $C$ energy ${ }^{7}$ which should be minimized by any stable solution. One may attempt to apply the scaling argument to the $C$ energy but one must check that the initial-value constraint ${ }^{3} R=16 \pi G T_{t}^{t}$ is invariant under the scaling $x^{\mu} \rightarrow \alpha x^{\mu}$ or equivalently $g_{\mu \nu} \rightarrow \alpha^{2} g_{\mu \nu}$, and this is only true if the potential term is identically zero. For global strings it does not seem to be easy to define a suitable notion of total energy per unit length.

Studies of the gravitational field of a U(1) global string have already encountered difficulties. While Harari and Sikivie $^{8}$ have calculated the metric of a global string using the weak-field approximation, Gregory ${ }^{5}$ has shown that, under certain assumptions about how the Higgs field approaches the potential minimum, there are no asymptotically well-behaved static cylindrically symmetric solutions. In Sec. II we derive the field and Einstein equations and prove in agreement with Ref. 5 that they have no regular asymptotic solutions with the Higgs field tending to its vacuum value. These results suggested that the spacetime could compactify. However, in Sec. III we prove a no-go theorem for regular compact warped product solutions for a general class of nonlinear $\sigma$ models, including static global strings as a special case, showing that they cannot compactify. The results of Sec. III also apply to $\sigma$-model strings, whose existence is discussed in Sec. IV. Section V is dedicated to explaining the relationship between Harari and Sikivie's weak-field approximation and singular solutions and we show that as the radial distance increases, the Higgs field will inevitably blow up. We also give a second asymptotically singular solution to the full equations, but this appears to have no relevance to cosmic strings.

\section{BASIC EQUATIONS AND NONEXISTENCE OF EVERYWHERE-WELL-DEFINED GLOBAL U(1) VORTICES}

Let us consider the model for a $U(1)$ global string, that is, a complex scalar field $\phi$ in a "Mexican hat" potential. The corresponding Lagrangian density is

$$
\mathcal{L}=-\frac{1}{2} g^{\mu \nu} \partial_{\mu} \phi \partial_{\nu} \bar{\phi}-\frac{\lambda}{4}\left(|\phi|^{2}-\eta^{2}\right)^{2},
$$


where $\lambda$ and $\eta^{2}$ are positive. The field and Einstein equations are

$$
\begin{aligned}
& \square \phi+\lambda \phi\left(\eta^{2}-|\phi|^{2}\right)=0, \\
& R_{\mu \nu}=8 \pi G\left(T_{\mu \nu}-\frac{1}{2} g_{\mu \nu} T\right),
\end{aligned}
$$

with the energy-momentum tensor given by

$$
T_{\mu \nu}=\partial_{\mu} \phi \partial_{\nu} \bar{\phi}-g_{\mu \nu} \mathcal{L} \text {. }
$$

We are interested in finding a static cylindrically symmetric string along the $x^{3}$ axis with boost invariance in the $x^{3}$ direction. This amounts to finding a solution of Eqs. (2.2) and (2.3) of the form

$$
\begin{aligned}
& d s^{2}=e^{A}\left[-d t^{2}+\left(d x^{3}\right)^{2}\right]+d \rho^{2}+\rho^{2} e^{B} d \theta^{2}, \\
& \phi=\eta f(\rho) e^{i \theta},
\end{aligned}
$$

where $A, B$, and $f$ are functions of $\rho$ only. Such a solution is usually called a vortex. Notice that in Eq. (2.6) we have taken the winding number of the string to be 1 .

Substituting Eqs. (2.5) and (2.6) into Eqs. (2.2) and 2.3) we get

$$
\begin{gathered}
f^{\prime \prime}+\left[A^{\prime}+\frac{B^{\prime}}{2}+\frac{1}{r}\right] f^{\prime}-\frac{f}{r^{2} e^{B}}+f\left(1-f^{2}\right)=0, \\
A^{\prime \prime}+A^{\prime 2}+\frac{A^{\prime} B^{\prime}}{2}+\frac{A^{\prime}}{r}=-4 \pi G \eta^{2}\left(f^{2}-1\right)^{2}, \\
2 A^{\prime \prime}+B^{\prime \prime}+A^{\prime 2}+\frac{B^{\prime 2}}{2}+\frac{2 B^{\prime}}{r} \\
=-4 \pi G \eta^{2}\left[4 f^{\prime 2}+\left(f^{2}-1\right)^{2}\right], \\
B^{\prime \prime}+\frac{B^{\prime 2}}{2}+A^{\prime} B^{\prime}+\frac{2 B^{\prime}}{r}+\frac{2 A^{\prime}}{r} \\
=-4 \pi G \eta^{2}\left[\frac{4 f^{2}}{r^{2} e^{B}}+\left(f^{2}-1\right)^{2}\right],
\end{gathered}
$$

the prime denoting differentiation with respect to $r$ $\equiv \sqrt{\lambda} \eta \rho$. We impose standard boundary conditions: $:^{3-5,8}$ the metric is locally flat at the $z$ axis, i.e.,

$$
A(0)=0, \quad e^{B} \sim 1 \text { as } r \rightarrow \infty,
$$

and

$$
f(0)=0, \quad f(r) \rightarrow 1 \text { as } r \rightarrow \infty .
$$

It is easy to show, ${ }^{3}$ using the Bianchi identities and the regularity of the metric at the axis, that Eq. (2.9) follows from Eqs. (2.7), (2.8), and (2.10). We further require one more condition, that the energy density

$$
T_{t}^{t}=\frac{\lambda \eta^{4}}{2}\left[f^{\prime 2}+\frac{f^{2}}{r^{2} e^{B}}+\frac{1}{2}\left(f^{2}-1\right)^{2}\right)
$$

vanishes as $r \rightarrow \infty$, which from a physical point of view is reasonable. ${ }^{3-5,8}$

As we have already mentioned, assuming that $f \rightarrow 1$ faster than some negative power of $r$, there are no asymptotically well-behaved static $U(1)$ global vortices. ${ }^{5}$ Here we briefly present a different proof, along the lines of that used by Garfinkle ${ }^{3}$ for local strings, without making any assumption about the way in which $f$ approaches 1 at infinity.

From Eqs. (2.7)-(2.10) it follows that

$$
\begin{aligned}
\theta_{1}\left(\theta_{2}-\frac{3}{4} \theta_{1}\right) & =8 \pi G H^{2} T_{r}^{r} \\
& =2 \pi G \lambda \eta^{4} H^{2}\left[f^{\prime 2}-\frac{f^{2}}{r^{2} e^{B}}+\left(f^{2}-1\right)^{2}\right),
\end{aligned}
$$

where $H \equiv r e^{A+B / 2}, \theta_{1} \equiv H A^{\prime}, \theta_{2} \equiv H^{\prime}$. Using analytical arguments one can prove that the right-hand side of this equation approaches zero as $r \rightarrow \infty$ (see the Appendix), and thus the only two possibilities for the asymptotic behavior of the metric are $\theta_{1} \rightarrow 0$ or $\theta_{2}-\frac{3}{4} \theta_{1} \rightarrow 0$. The first case gives an asymptotically conical metric but this cannot correspond to the physical solution. This can be seen by evaluating the deficit angle $\delta$. The Gauss-Bonnet theorem yields

$$
\delta \geq \frac{16 \pi^{2} G}{\eta^{4}} \int_{0}^{\infty} d r r e^{B / 2} T_{t}^{t},
$$

which diverges logarithmically. The second case, $\theta_{2}-\frac{3}{4} \theta_{1} \rightarrow 0$, is a Kasner metric with equivalent asymptotics to those of Melvin's magnetic universe. ${ }^{9}$ However, this does not describe a physical solution either since for this metric the metric coefficient $r^{2} e^{B}$ goes to zero as $r \rightarrow \infty$ and then the energy density $T_{t}^{t}$ diverges at infinity, contradicting our previous assumptions.

These results confirm that there are no well-behaved asymptotic solutions to Eqs. (2.7)-(2.10). Both the solutions we have ruled out have the common feature that they correspond in some sense to a closing up of spacetime. However, in the next section we further prove that there are no regular static spatially compact solutions.

\section{A NO-GO THEOREM FOR STATIC COMPACT GLOBAL STRINGS}

The question of compactification is of interest in a variety of contexts, and so we derive a no-go theorem for a general class of nonlinear $\sigma$ models in $d$-dimensional Euclidean spacetime. The static global string will appear as a special case after we have used the time independence of the equations to perform a Wick rotation. The model we consider has the Lagrangian

$$
\mathcal{L}=-\frac{1}{2} G_{A B}(\phi) g^{\mu v} \partial_{\mu} \phi^{A} \partial_{\nu} \phi^{B}-V(\phi),
$$

where $V(\phi)$ is any non-negative potential. Notice that the $\mathrm{U}(1)$ global string is a particular case. In Eq. (3.1) $g_{\mu v}$ and $G_{A B}$ are the metrics on the spacetime manifold $M_{d}$ and the target space, respectively. The former is assumed to have the form

$$
\begin{aligned}
& d s^{2}=W\left(x^{l}\right) \delta_{a b} d y^{a} d y^{b}+h_{m n}\left(x^{l}\right) d x^{m} d x^{n}, \\
& \left\{M_{d}, g_{\mu \nu}\right\}=\left\{N_{d-D}, \delta_{a b} W\right\} \times\left\{P_{D}, h_{m n}\right\}, \\
& \mu, v=0,1, \ldots, d-1, \quad a, b=0,1, \ldots, d-D-1, \\
& m, n=d-D, \ldots, d-1,
\end{aligned}
$$


where the metric $g_{\mu \nu}$ is of Euclidean signature. It has $d-D$ hypersurface-orthogonal Killing vectors $\partial / \partial y^{a}$. The energy-momentum tensor for this model is

$$
T_{\mu \nu}=G_{A B}(\phi) \partial_{\mu} \phi^{A} \partial_{\nu} \phi^{B}+g_{\mu \nu} \mathcal{L},
$$

which together with the Einstein equation (2.3) gives

$$
R_{\mu \nu}=8 \pi G\left[G_{A B}(\phi) \partial_{\mu} \phi^{A} \partial_{\nu} \phi^{B}+\frac{2}{d-2} g_{\mu \nu} V(\phi)\right] \text {. }
$$

Under these assumptions the following theorem holds: if the Ricci tensor is non-negative, $W \neq 0$, and $\left(P_{D}, h_{m n}\right)$ is regular and compact, then $W$ is constant and $R_{\mu v} K^{\mu} K^{v}=0$ for $K^{\mu}$ any of the above Killing vectors.

To prove this we introduce the twist three-form $\omega_{\mu \nu \lambda}=K_{[\mu} \nabla_{v} K_{\lambda]}$ and note that, for any of the $d-D$ Killing vectors, $W=K_{\mu} K^{\mu}$. After some simple algebra we obtain

$$
\omega^{2}=-\frac{1}{6}(\nabla W)^{2}+\frac{1}{3} W\left(\nabla_{\mu} K_{v}\right)\left(\nabla^{\mu} K^{v}\right) .
$$

On the other hand, the Bianchi identities give

$$
\square W=2\left(\nabla_{\mu} K_{v}\right)\left(\nabla^{\mu} K^{v}\right)-2 R_{\mu \nu} K^{\mu} K^{v} .
$$

From these two equations we get

$$
\nabla\left[\frac{1}{W} \nabla W\right]=6 \frac{\omega^{2}}{W^{2}}-2 \frac{R_{\mu v} K^{\mu} K^{v}}{W} .
$$

Since the $K^{\mu}$ are hypersurface orthogonal we have that $\omega_{\mu \nu \lambda}=0$. Integrating now over $\boldsymbol{M}_{d}$ - the $y$ independence of the metric reduces this to an integration over the compact manifold $P_{D}$ - the total derivative term vanishes and we are left with

$$
\int d^{D} x \sqrt{h} W^{(d-D-2) / 2} R_{\mu v} K^{\mu} K^{v}=0 .
$$

This implies that $R_{\mu \nu} K^{\mu} K^{v}=0$ throughout $M_{d}$ since $R_{\mu \nu} \geq 0$. To see that $W$ is a constant we again use Eqs. (3.5) and (3.6) to obtain

$$
W R_{\mu \nu} K^{\mu} K^{v}-3 \omega^{2}+\frac{1}{2} \nabla(W \nabla W)-(\nabla W)^{2}=0 .
$$

We now integrate and use $\omega^{2}=0=R_{\mu \nu} K^{\mu} K^{v}$ to get

$$
\int d^{D} x \sqrt{h} W^{(d-D) / 2}(\nabla W)^{2}=0,
$$

from which it follows that $W$ is constant.

This result gives the following form for the Ricci tensor:

$$
R_{\mu \nu}=\left(\begin{array}{ll}
0_{d-D} & \\
& R_{m n}
\end{array}\right), \quad R_{m n} \geq 0 .
$$

Equating Eqs. (3.4) and (3.7) we have that the potential must be identically zero, $V(\phi)=0$. Having shown that $V(\phi)=0$, the fields $\phi^{A}$ are now harmonic maps from $M_{d}$ into the target space. Finding the possible $\phi^{A}$ is then a problem of harmonic maps. ${ }^{10}$

In the case of four spacetime dimensions, Eqs. (3.4) and (3.7) also imply that $\phi^{A}$ is independent of $y^{a}$ provided that $G_{A B}$ is Riemannian. We are thus left with $R_{m n}=G_{A B}(\phi) \partial_{m} \phi^{A} \partial_{n} \phi^{B}$. For the case $D=2, R_{m n}$ $=\kappa h_{m n}$, where $\kappa$ is the Gauss curvature. Integrating we obtain

$$
\int d^{2} x \sqrt{h} \kappa=\int d^{2} x \sqrt{h} h^{m n} G_{A B}(\phi) \partial_{m} \phi^{A} \partial_{n} \phi^{B} \geq 0 .
$$

The left-hand side is equal to $4 \pi(1-g)$, where $g$ is the genus of $P_{2}$, and we deduce that $g=0$ or $g=1$. The latter does not correspond to a compact solution since it requires $\kappa$ to vanish everywhere. We conclude that the only possible topology for $P_{2}$ is that of a two-sphere. Furthermore, if the target space is flat then $\phi^{A}=$ const (see Sec. IV below).

Let us see how these results apply to the global string of Eq. (2.1). The Lagrangian density is of the form (3.1), with $V(\phi)=(\lambda / 4)\left(|\phi|^{2}-\eta^{2}\right)^{2}$. The target space has dimension 2, metric $G_{A B}=\delta_{A B}$, and coordinates $\phi^{1}=\frac{1}{2}(\phi+\bar{\phi}), \phi^{2}=(i / 2)(\bar{\phi}-\phi)$. We perform a Wick rotation, $t=-i \tau$, and work with Euclidean signature since the field equations (2.7)-(2.10) are time independent. The spacetime metric is then

$$
d s^{2}=e^{A}\left(d \tau^{2}+d z^{2}\right)+d r^{2}+r^{2} e^{B} d \theta^{2},
$$

which is of the type (3.2) and has a non-negative Ricci tensor. According to the above result the existence of an $(r, \theta)$ compact solution requires $V(\phi)=0$ and $A$ and $\phi$ to be constants, and so the spacetime does not contain a global string.

\section{IV. $\sigma$-MODEL STRINGS}

In general, nonlinear $\sigma$ models also admit stringlike solutions. To see this let us consider the Lagrangian density of Eq. (3.1) with a zero potential:

$$
\mathcal{L}=-\frac{1}{2} \xi^{2} G_{A B}(\phi) g^{\mu v} \partial_{\mu} \phi^{A} \partial_{\nu} \phi^{B},
$$

where we have introduced a coupling constant $\xi$ with dimensions of (length) ${ }^{-1}$ since we are taking the fields $\phi$ to be dimensionless. We are interested in stringlike solutions, that is, static solutions with an axial Killing vector so that

$$
d s^{2}=e^{2 \Sigma}\left[-d t^{2}+\left(d x^{3}\right)^{2}\right]+e^{2 \Psi} d z d \bar{z},
$$

where $(z, \bar{z})$ is a complex local coordinate patch and $\Sigma$ and $\Psi$ are functions of $z$ and $\bar{z}$ only.

Spatially compact solutions of the type (4.1) are governed by the results of the previous section. Here we shall discuss the existence of $\sigma$-model strings for noncompact spacetimes. We will restrict ourselves to the case of two-dimensional target spaces, for which the metric can always be written as $G_{A B}=\Omega^{2} \delta_{A B}$ for suitable $\Omega$.

We seek solutions of the field and Einstein equations with metric of the form (4.1) and for which the field $\phi$ depends only on $z$ and $\bar{z}$. The $\phi$ field equation is

$$
\begin{aligned}
& \partial \bar{\partial} \phi+(\partial \Sigma)(\bar{\partial} \phi)+(\bar{\partial} \Sigma)(\partial \phi) \\
& +2\left[(\partial \phi)(\bar{\partial} \phi) \frac{\partial \ln \Omega}{\partial \phi}+(\partial \bar{\phi})(\bar{\partial} \bar{\phi}) \frac{\partial \ln \Omega}{\partial \bar{\phi}}\right]=0,
\end{aligned}
$$

where $\partial$ and $\bar{\partial}$ stand for $\partial / \partial z$ and $\partial / \partial \bar{z}$, respectively.

Now the energy density is minimized by fields satisfy- 
ing Bogomol'nyi-type equations, which are solved by any holomorphic function $\phi$ (Ref. 11). Thus configurations of minimal energy satisfy $\bar{\partial} \phi=0$, and for these the field equation simplifies to

$$
(\bar{\partial} \Sigma)(\partial \phi)=0 \text {, }
$$

which has two solutions. The first is $\partial \phi=0$, which, together with $\bar{\partial} \phi=0$, is equivalent to the statement that $\phi=$ const. The other is $\bar{\partial} \Sigma=0$, from which it follows that $\Sigma=$ const since $\Sigma$ is real. For $\phi=$ const the solutions of type (4.1) to the Einstein equations are no more than vacuum solutions with one Killing vector and have been widely studied. ${ }^{9}$ We shall concentrate on the more interesting possibility $\Sigma=$ const, which is equivalent to $\Sigma=0$ since this amounts to a rescaling of $t$ and $x^{3}$ in (4.1).

The physical meaning of solutions with $\Sigma=0$ is clear: the problem of stringlike solutions of type (4.1) has been reduced to that of finding static solitons in $2+1$ dimensions, or equivalently instantons in two dimensions. The Bogomol'nyi equations ensure that any solution will correspond to a stable soliton, subject to the assumption that the holomorphic function tends to a fixed map at infinity.
For $\Sigma=0$ the only nontrivial Einstein equation is

$$
\partial \bar{\partial} \Psi=-2 \pi G \xi^{2} \Omega^{2}(\partial \phi)(\bar{\partial} \bar{\phi}) .
$$

In Ref. 11 some solutions to this equation are given for the case in which the target space is $C^{1}$ with metric $\Omega=2 /(1+\phi \bar{\phi})$. For $\phi=\beta / z$, with $\beta$ a constant, $\Psi$ takes the form

$$
\Psi=-8 \pi G \xi^{2} \ln \left(|z|^{2}+|\beta|^{2}\right)
$$

For large values of $z$ one can make the change $(1-\alpha) \rho$ $=|z|^{(1-\alpha)}$, with $\alpha \equiv 8 \pi G \xi^{2}$, which gives the following asymptotic form for the metric (4.1), (4.3):

$$
d s^{2} \sim-d t^{2}+\left(d x^{3}\right)^{2}+d \theta^{2}+\alpha^{2} \rho^{2} d \rho^{2} .
$$

Thus the metric is asymptotically conical with deficit angle $32 \pi^{2} G \xi^{2}$.

Independently of whether one is looking for compact or noncompact solutions some restrictions can be obtained on the geometry of the target space when looking for stringlike solutions. In Ref. 10 the following identity is derived:

$$
\int \sqrt{-g} d^{4} x \mathcal{L}_{; \mu}^{; \mu}=\int \sqrt{-g} d^{4} x \phi_{A \mu ; \nu} \phi^{A \mu ; v}-\int \sqrt{-g} d^{4} x\left(R_{A B C D} \phi^{A \mu} \phi_{\mu}^{B} \phi^{C v} \phi_{\nu}^{D}-R_{\mu \nu} \phi^{A \mu} \phi_{A}^{v}\right)
$$

where $\phi_{A \mu} \equiv \partial_{\mu} \phi_{A}$ and the spacetime metric is assumed to be regular. Typically, a harmonic map corresponding to a soliton in $2+1$ dimensions will tend to a fixed map at least as $1 / z$ as the proper length goes to infinity. For such behavior, the boundary term containing the Lagrangian density will vanish.

If the Ricci tensor of the spacetime manifold is nonnegative, we can exclude target spaces with negativesemidefinite curvature $R_{A B C D}$ as follows. In order to satisfy Eq. (4.4) the following identities must hold everywhere:

$$
\begin{aligned}
& \phi_{A \mu ; v}=0, \\
& R_{A B C D} \phi^{A \mu} \phi_{\mu}^{B} \phi^{C v} \phi_{v}^{D}=0,
\end{aligned}
$$

and

$$
R_{\mu \nu} \phi^{A \mu} \phi_{A}^{\nu}=0 \text {. }
$$

Now, since the target space is two dimensional, Eq. (4.7) reduces to

$$
(\partial \phi)(\bar{\partial} \bar{\phi})=0 \text {, }
$$

from which it follows that $\phi=$ const, which is again the trivial case.

Naively one could think of repeating the construction of stringlike solutions for the $\mathrm{CP}^{1}$ model above for spaces of negative curvature. Although one is able to construct such solutions, they correspond to singular spacetime geometries. Consider, for example, the unit disc with Poincaré metric, $\Omega=2 /(1-\phi \bar{\phi})$, and take $\phi=z / \beta$ with $|z|<|\beta|$. The solution to Eq. (4.2) is, then,

$$
\Psi=8 \pi G \xi^{2} \ln \left(|z|^{2}-|\beta|^{2}\right),
$$

for which the scalar curvature is $R=4 \alpha /\left(|\beta|^{2}\right.$ $\left.-|z|^{2}\right)^{2(1+\alpha)}$, where $\alpha \equiv 8 \pi G \xi^{2}$. $R$ takes arbitrarily large values as $|z| \rightarrow|\beta|$ and so the transverse spatial sections become more and more curved as $|z| \rightarrow|\beta|$. Notice that such sections are topologically noncompact but they have finite volume since

$$
\int_{|z| \leq|\beta|} e^{2 \Psi} d^{2} z=\frac{\pi}{1+2 \alpha}|\beta|^{2(1+2 \alpha)} .
$$

If we visualize one of these sections by embedding it isometrically in a three-dimensional Euclidean space we obtain a teardrop of the kind derived by Gell-Mann and Zwiebach ${ }^{12}$ in the context of higher-dimensional supergravity theories.

\section{SINGULAR SOLUTIONS AND THEIR PHYSICAL SIGNIFICANCE}

The system of Eqs. (2.7)-(2.10) does at least admit a solution in the weak-field approximation. ${ }^{8}$ By studying Eq. (2.7) we shall show that for the physical solution picked out by this approximation the Higgs field blows up. The corresponding configuration turns out to be singular at large radius (the spacetime will close up at a curvature singularity ${ }^{13}$ ), and this result is thus compatible with those of Secs. II and III, as it does not satisfy condition (2.11). In addition we present a second singular solution, for which exact asymptotics are known, but argue that it does not correspond to a string configuration.

Harari and Sikivie, using the weak-field approximation and assuming that $f \equiv 1$, found the following form for the 
metric

$$
e^{B}=1-2 \mu \ln r+c, \quad e^{A}=1-\mu \ln r,
$$

where $\mu=4 \pi G \eta^{2}$. This approximation turns out to be valid out to $\rho<<\rho_{c}$ where

$$
\rho_{c} \sim \frac{1}{\sqrt{\lambda} \eta} \exp \left(\frac{1}{8 \pi G \eta^{2}}\right)
$$

is much larger than the present horizon scale.

Consider now Eq. (2.7),

$$
f^{\prime \prime}+\left[A^{\prime}+\frac{B^{\prime}}{2}+\frac{1}{r}\right] f^{\prime}-\frac{f}{r^{2} e^{B}}+f\left(1-f^{2}\right)=0 .
$$

We may regard this as the equation for a particle in an inverted Mexican hat potential, $-V(\phi)$, under a frictional force (since $A^{\prime}+\frac{1}{2} B^{\prime}+1 / r>0$ ) and a force $f / r^{2} e^{B}$. When $f$ is close to 1 , in the sense of the weak-field approximation, the frictional force is negligible, and the constancy of $f$ implies that

$$
-\frac{1}{r^{2} e^{B}} \simeq 2 \epsilon \text { where } f=1+\epsilon
$$

The existence of a family of flat-space solutions [i.e., solutions to (2.7) when $e^{B}=1$ and $e^{A}=1$ ] shows that if the force is $1 / r^{2}$, the "particle" will just reach the peak. However, the weak-field approximation predicts that $e^{-B}>1$ near $f=1$ and that $1 / r^{2} e^{B}$ will begin to increase as $r$ approaches $r_{c}$.

This qualitative behavior will remain valid if we relax the weak-field approximation, demand only that $\epsilon$ be small, and solve the resulting equations in $A$ and $B$. We see from such an analysis that the force indeed exceeds $1 / r^{2}$ and that the friction is less than the flat-space value. Thus as we approach $r_{c}, \epsilon$ will reach zero. From this point on, the behavior of the system is evident: $f$ will rapidly grow, and such growth will drive $e^{A}$ and $e^{B}$ even more rapidly toward zero. The result is a curvature singularity at the point of nonregular compactification.

A study of the cylindrically symmetric vacuum Einstein equations with definite cosmological constant yields a Kasner metric:

$$
\begin{aligned}
& e^{A}=\cos \left[\left(\frac{3 \Lambda}{4}\right)^{1 / 2} \rho\right], \\
& \rho^{2} e^{B}=\frac{4}{3 \Lambda} \sin ^{2}\left[\left(\frac{3 \Lambda}{4}\right]^{1 / 2} \rho\right] \cos ^{-2 / 3}\left[\left(\frac{3 \Lambda}{4}\right)^{1 / 2} \rho\right] .
\end{aligned}
$$

This metric is singular at $\rho_{0}=\pi / \sqrt{3 \Lambda}$ and corresponds to a solution to Eqs. (2.7)-(2.10) with $f \equiv 0$, and $\Lambda=2 \pi G \lambda \eta^{4}$. This is also, however, an asymptotic solution to the equations for $f \rightarrow 1$ and $f^{\prime} \rightarrow 0$ as $\rho \rightarrow \rho_{0}$. While this solution would appear to have some physical significance, since the Higgs field approaches an energy minimum, a numerical analysis has shown that if we evolve $f$ back from $\rho_{0}$ it will grow rather than decrease.
This demonstrates that we cannot match this solution with one satisfying our imposed boundary conditions at the origin, since if $f>1$ then $f$ cannot have a turning point.

\section{CONCLUSIONS}

The purpose of this paper was to see whether one can talk meaningfully about the gravitational field of a static isolated global string. There are just two boost-invariant vacuum metrics with whole-cylinder symmetry. One corresponds to an asymptotically flat conical metric, the second to an asymptotically Melvin-type metric. In the first case the transverse $(\rho, \theta)$ space opens out to form a flat cone with finite deficit angle $\delta<2 \pi$. In the second it closes up but never quite "compactifies"- the $(\rho, \theta)$ surface resembles an infinitely long onion.

The conical metric corresponds to the gravitational field of a local string. The onion metric corresponds to the gravitational field of a Melvin flux tube. Before undertaking this investigation we had thought that the onion metric might be relevant for the gravitational field of a global string. In fact this is not so. We have shown that there is no universal well-behaved form of the metric to which all static global strings tend. We have also proved that there are no static solutions in which the transverse $(\rho, \theta)$ directions are compactified. In fact the gravitational field of a global string is singular at infinity and our results show that the Higgs field also blows up at infinity.

From a physical point of view this singularity is probably unimportant. The distance away from the string at which its effects would be felt is of the order of

$$
\rho_{c} \sim \frac{1}{\sqrt{\lambda} \eta} \exp \left(\frac{1}{8 \pi G \eta^{2}}\right)
$$

For realistic values of $\lambda$ and $\eta$ this distance is enormous. It is far larger than the horizon size at present. At such large distances it is unreasonable to consider only the field of the string alone. The other matter in the Universe would completely modify the metric. However, a consequence of the impossibility of finding a welldefined asymptotic field of a global string is that, just as in flat spacetime, one cannot talk about the total energy per unit length of a global string. This is quite different from the local case, where the deficit angle $\delta$ or equivalently Thorne's energy play this role.

The situation for nonlinear $\sigma$-model strings is somewhat different. As in the global case, there are no static solutions with compact transverse $(r, \theta)$ sections. But now there are regular everywhere-well-defined metrics with conical asymptotics. Finally, one also has solutions in which the spacetime closes up at a singularity.

Note added in proof. The critical radius $\rho_{c}$ is an extremely sensitive function of $\eta$. As has recently been pointed out in a paper we received after the completion of this work (Cohen and Kaplan ${ }^{14}$ ), if $\eta \simeq 2 \times 10^{17} \mathrm{GeV}$ (which is rather high for grand unified theory strings) $\rho_{c} \simeq 10^{28} \mathrm{~cm}$, the horizon radius. 


\section{ACKNOWLEDGMENTS}

We would like to thank Tanmay Vachaspati and Ruth Gregory for valuable discussions. F.R.R. is indebted to the Department of Applied Mathematics and Theoretical Physics for their kind hospitality in Cambridge and to the Spanish Ministry of Education and Science for support.

\section{APPENDIX}

Here we prove that the right-hand side of Eq. (2.12), $\theta_{1}\left(\theta_{2}-\frac{3}{4} \theta_{1}\right)=8 \pi H^{2} T_{r}^{r}$, tends to zero at large distances. In the case of local strings, ${ }^{3}$ certain assumptions about the way in which $f \rightarrow 1$ are used to prove that $\lim _{r \rightarrow \infty} H^{2} T_{r}^{r}=0$. We present a proof for the global string case where we assume only that the metric is well behaved and that $f \rightarrow 1$.

From Eqs. (2.7)-(2.10) we get that

$$
H^{\prime \prime}=4 \pi H\left[-\frac{4 f^{2}}{r^{2} e^{B}}-12\left(f^{2}-1\right)^{2}\right]
$$

and so, using the appropriate boundary conditions on the axis,

$$
H^{\prime}=1-\int_{0}^{r} 4 \pi H\left[\frac{4 f^{2}}{r^{\prime 2} e^{B}}+12\left(f^{2}-1\right)^{2}\right] d r^{\prime} .
$$

Because $H$ remains positive, $H^{\prime}$ is bounded below, and so we have

$$
\int_{0}^{r} H\left[\frac{4 f^{2}}{r^{\prime 2} e^{B}}+12\left(f^{2}-1\right)^{2}\right) d r^{\prime}<\infty,
$$

which implies that $H f^{2} / r^{2} e^{B}$ and $H\left(f^{2}-1\right)^{2}$ fall off as $r^{a}$, where $a<-1$. Now

$$
H=r-\int_{0}^{r} d r^{\prime} \int_{0}^{r^{\prime}} 4 \pi H\left[\frac{4 f^{2}}{r^{\prime \prime 2} e^{B}}+12\left(f^{2}-1\right)^{2}\right] d r^{\prime \prime}
$$

and so, since $H$ is positive and hence less than $r$, asymptotically $H^{2} f^{2} / r^{2} e^{B}$ and $H^{2}\left(f^{2}-1\right)^{2}$ will behave as $r^{b}$, where $b<0$. Hence,

$$
\lim _{r \rightarrow \infty} \frac{H^{2} f^{2}}{r^{2} e^{B}}=0=\lim _{r \rightarrow \infty} H^{2}\left(f^{2}-1\right)^{2}
$$

It remains to show that $\lim _{r \rightarrow \infty} H^{2} f^{\prime 2}=0$. Now Eq. (2.7) tells us that if at some finite radius $f=1$, then $f$ blows up as $r \rightarrow \infty$. Hence $f<1$ for all $r$ and, since we cannot have oscillatory behavior, there will be some $r_{0}$ after which $f$ will tend monotonically to 1 , so that $f^{\prime 2} \rightarrow 0$ faster than $\left(f^{2}-1\right)^{2} \rightarrow 0$.
${ }^{*}$ On leave of absence from Departamento de Física Teórica, Universidad Complutense, Madrid, Spain.

${ }^{1}$ A. Vilenkin, Phys. Rep. 121, 263 (1985).

${ }^{2}$ H. B. Nielsen and P. Olesen, Nucl. Phys. B61, 45 (1973).

${ }^{3}$ D. Garfinkle, Phys. Rev. D 32, 1323 (1985).

${ }^{4}$ P. Laguna-Castillo and R. Matzner, Phys. Rev. D 36, 3663 (1987).

${ }^{5}$ R. Gregory, Phys. Rev. Lett. 59, 740 (1987).

${ }^{6}$ G. H. Derrick, J. Math. Phys. 5, 1252 (1964).

${ }^{7}$ K. Thorne, Phys. Rev. 138, B251 (1965).
${ }^{8}$ D. Harari and P. Sikivie, Phys. Rev. D 37, 3448 (1988).

${ }^{9}$ D. Kramer, H. Stephani, M. MacCallum, and E. Herlt, Exact Solutions of Einstein's Field Equations (VEB Deutscher Verlag der Wissenschaften, Berlin, 1980).

${ }^{10}$ C. W. Misner, Phys. Rev. D 18, 4510 (1978).

${ }^{11}$ A. Comtet and G. W. Gibbons, Nucl. Phys. B299, 719 (1988).

${ }^{12}$ M. Gell-Mann and B. Zwiebach, Phys. Lett. 147B, 111 (1984).

${ }^{13}$ R. Gregory, Phys. Lett. B 215, 663 (1988).

${ }^{14}$ A. D. Cohen and D. B. Kaplan, Phys. Lett. B 215, 67 (1988). 